\title{
Design of Portable Otoacoustic Emission Detecting System Evoked by Swept-tone
}

\author{
Huang He-Qing \\ Chongqing College of Electronic engineering, Chongqing 401331,China
}

Keywords: Swept-tone otoacoustic emission, LabView, NI-6218, Hearing loss

\begin{abstract}
Otoacoustic emission(OAE) is a sound energy generated by human cochlea. Since it is closely related to the functional status of inner ear, the study of OAE is one of most important process in hearing physiology. In view of great shortcoming of the present OAE detection, such as single feature, ponderous, costliness and so on, a portable system of OAE detecting evoked by swept-tone is introduced in this paper. It also presents the hardware design and detection algorithm of this system in detail. Experimental results show that the portable system has such advantage as convenient operation, small volume and price cheap, etc. Hence, it possesses a strong practicability and beneficial in the development of a new type of OAE instrument.
\end{abstract}

\section{Introduction}

Otoacoustic emission is one of the most important discoveries in physiology over the past 3 decades, witch uncovers that the ears can emit sound wave besides receiving sound. It provides a safe, simple and sensitive method to detect the degree of hearing damage. The primitive Otoacoustic emission detectors tend to be large in size with simple functions and can not meet the needs of application. Over the past several years, many researches on Otoacoustic emission have been carried out in physiology field, but the design and manufacture of electronic detectors fall behind. So we propose a scheme in this paper to design a type of portable otoacoustic emission detector, which employs swept-tone signals instead of pure sounds to detect OAE. It will greatly improve the detecting efficiency with smaller size, lower power consumption and conveniences to adjust.

\section{Design Scheme}

This system, as is shown in figure 1, is comprised of the processes of synthesis, adjustment, playing and sampling of stimulation-sound, power component and communication control. The synthesis and adjustment of stimulation-sound are completed by the software of host. The playing and sampling of stimulation-sound include a data collection circuit, a amplifier and an optical probe. The output of the data sampling circuit is converted into sound pressure by a loudspeaker, then is played out by a sound probe put in the user's outer ear. A mini microphone embedded in the sound probe collects the feedback signals in the user's ear and converts the signals into electric voltages which are amplified and sampled by the data collection circuit. The power component supplies various electric voltages for the system components. The function of communication control component is mainly to coordinate and supervise the works of all other components. 


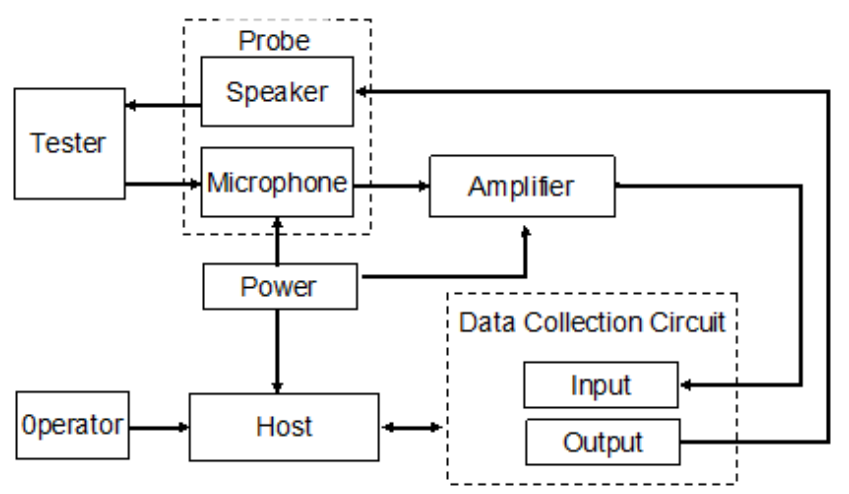

Figure 1 Structure of portable OAE detection system

In the procedure of otoacoustic emission detection, each experiment need to sample the feedback signals and play the stimulation sound several times, and the synchronization is essential. In order to evoke the otoacoustic emission with a broad frequency band in a short time, the accuracy of data sampling circuit is very important because of the low voltage of signals. We select USB-6218 as the core chip to fulfill the high sample rate with $250 \mathrm{KS} / \mathrm{s}$ and the high sample accuracy with 16bit, which make it possible to collect the wick otoacoustic emission signals. This circuit provides the A/D and D/A converts, 32 analogy inputs and 2 analogy outputs. Each channel can select an optimal sample accuracy by adjusting the rang of electric voltage and currency. With the digital evocation, this system can meet the strict requirement of synchronization between sampling and playing of otoacoustic emission signals.

Generally, the swept-tone signal voltage got from microphone is in the rage of $1 \mathrm{mv}$ to $20 \mathrm{mv}$, and the rage of frequency is between $500 \mathrm{~Hz}$ to $10 \mathrm{KHz}$ with a great amount of background noise and disturbance. So the amplifying circuit is introduced to boost and filter the feedback signals. This circuit is comprised of preamplifier, filter and main amplifier. The preamplifier is designed with AD620 to ensure the signals with high common mode rejection ratio. The UAF42 chip is employed in filter component to achieve a pass band between $0.4 \mathrm{KHz}$ to $12 \mathrm{KHz}$, since the valuable signals reside in $0.5 \mathrm{KHz}$ to $10 \mathrm{KHz}$.

The amplifying times varies under different conditions, so the main amplifying circuit employs TLC2274 and ADG804 multiplexing switch to achieve a dynamic amplifying function, the structure of which is shown as figure 2 . The resistor of $R 1$ to $R 4$ induces the gain of $0 \mathrm{~dB}, 10 \mathrm{~dB}, 20 \mathrm{~dB}$ and $40 \mathrm{~dB}$ respectively.

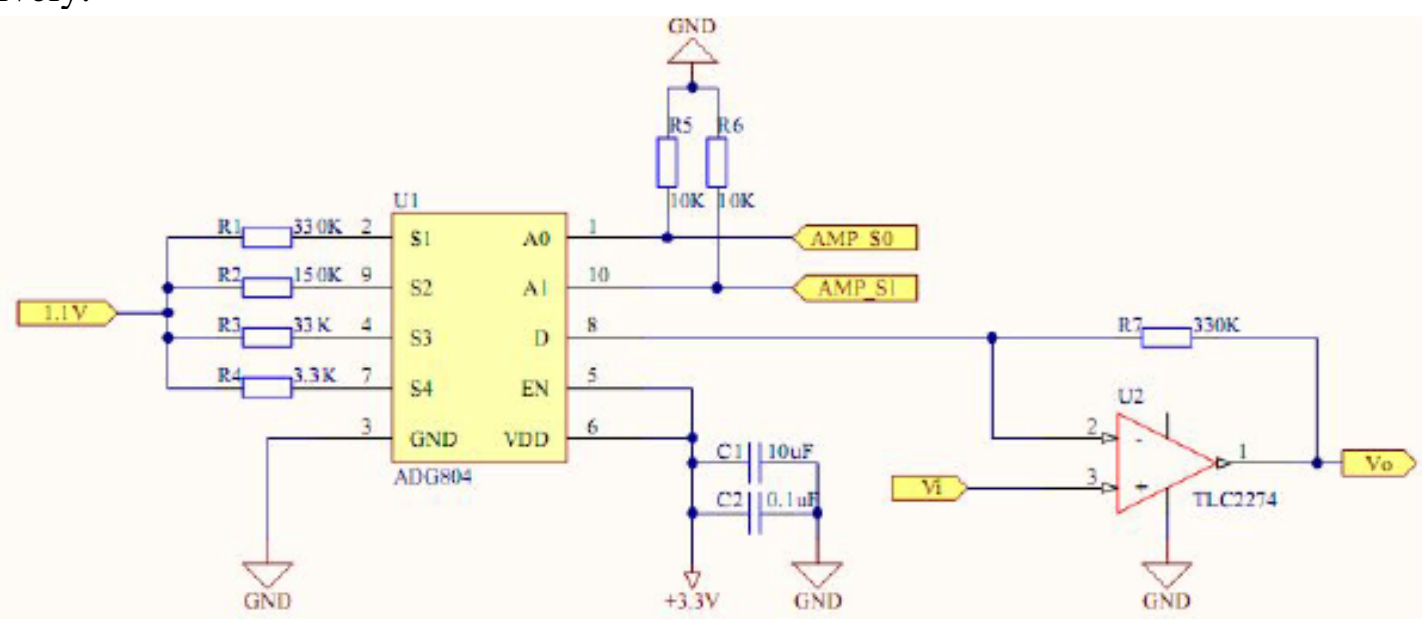

Figure 2 Main amplifying circuit diagram

After a simple calculation, we can the amplifying times as follows:

$$
A=20 \lg \frac{V_{0}}{V_{i}}=20 \lg \left(1+\frac{R_{7}}{R_{x}}\right)
$$

This system employs the detection method of DPOAE with swept-tone and DPOAE with pure sound, which is developed on the basis of LabView platform. The software structure can be divided 
into 4 parts: station detection of sound probe, stimulation sound synthesizing and adjustment, signals detection supervision and signals storage. Firstly, I/O selection of data sampling circuit and channels synchronization are initialized, and all actions on I/O are strictly synchronized by the rising edge of clock signal to ensure that the stimulation sound is played as soon as the OAE signals are sampled. After the selection of detection type, the program produces a stimulation sound and plays it through speaker system, then samples the feedback signals and put them into storage. We can use Matlab to analyze the signals to judge the damage level of ear.

A reasonable position of sound probe is essential to get the valid OAE signals because of irregular shape of ear. So it needs to detect the position of sound probe before this system begins to sample OAE signals. We should alter the probe position until the frequency field of signals converted by FFT does not overlap and the background noise does not disturb the valid signals between $500 \mathrm{~Hz}$ to $10 \mathrm{KHz}$.

We use LabView to generate pure sound with fix frequency and Chirp signals with linearly increasing frequency. It is proved by experiments that the frequency responses are different on various speaker devices with the same currency input. As a result, we adjust the stimulation sound amplitude before each detection to avoid the above problems influence detection results. According to the sound pressure feedback technology, we complete adjustment by minimizing the sound pressure feedback error and the difference between stimulation sound and expected response.

\section{Simulation and Experiment}

This experiment is carried out in 9 volunteers with DPOAE detection, and the parameters are shown in table 1 . The frequency and amplitude of main stimulation sound are represented as $f_{1}$ and $L_{1}$ respectively, and the frequency and amplitude of sub-stimulation sound are represented as $f_{2}$ and $L_{2}$ respectively. Here, symbol $\mathrm{T}$ is the time length of signal, ST means sampling times and FR means frequency resolution.

Table 1 Stimulus parameters of DPOAE detection

\begin{tabular}{|lllllllllll|}
\hline & $\mathrm{f}_{1}$ & $\mathrm{~L}_{1}$ & $\mathrm{f}_{2} / \mathrm{f}_{1}$ & $\mathrm{~L}_{1}-\mathrm{L}_{2}$ & $\mathrm{~T}$ & $\mathrm{ST}$ & $\mathrm{FR}$ \\
\hline Pure sound oriented & $1-4 \mathrm{KHz}$ & $55 \mathrm{~dB}$ & 1.2 & $5 \mathrm{~dB}$ & $1 \mathrm{~s}$ & 10 & $25 \mathrm{~Hz}$ \\
\hline Swept-tone oriented & $0.5-10 \mathrm{KHz}$ & $55 \mathrm{~dB}$ & 1.2 & $5 \mathrm{~dB}$ & $1 \mathrm{~s}$ & 20 & $1 \mathrm{~Hz}$ \\
\hline
\end{tabular}

DPOAE detection is tested in a group of people with listening damage, but we single out one case here to illustrate the experiment. It is carried out with $\mathrm{f}_{1}$ equals $1475 \mathrm{~Hz}$. We sample the signals 10 times and average them, then get the wave in frequency field by FFT conversion as shown in figure 3(a). The result implies that DPOAE signals with frequency of $1180 \mathrm{~Hz}$ are produced successfully under the condition that main stimulus $\mathrm{f}_{1}$ equals $1475 \mathrm{~Hz}$ and sub-stimulus $\mathrm{f}_{2}$ equals $1770 \mathrm{~Hz}$. After a simple conversion, we can get a amplitude curve as shown in figure 3(b), which shows 3 obvious drops at the frequencies of $1.7 \mathrm{KHz}, 3.8 \mathrm{KHz}$ and $6.7 \mathrm{KHz}$. So we can make a conclusion that this people has some listening damages at about these three frequency points.
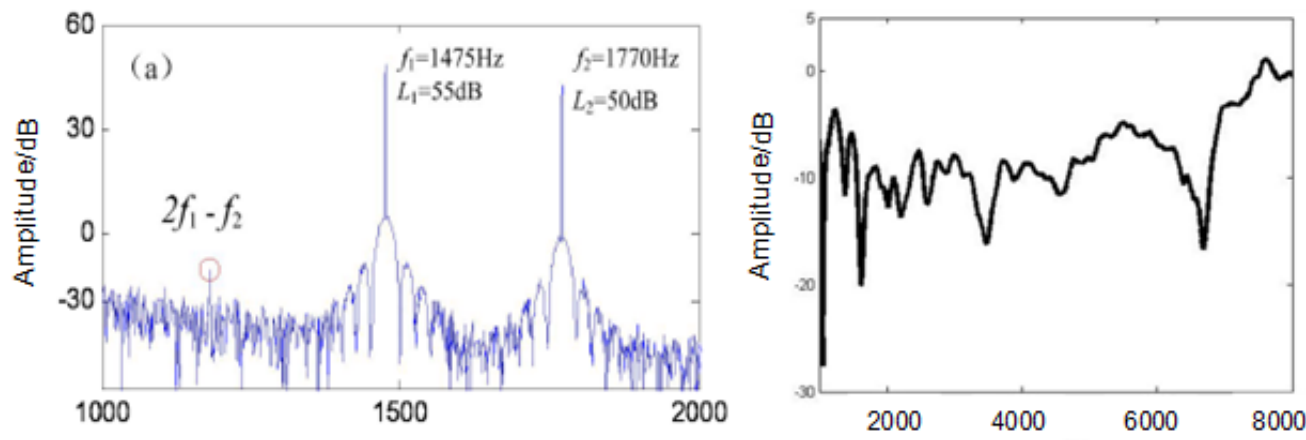

Fig 3(a) DPOAE response in frequency Fig 3(b) DPOAE amplitude curve

Figure 3 Results of DPOAE evoked by swept-tone 


\section{Conclusion}

We propose a new design for portable otoacoustic emission detecting system evoked by swept-tone on the basis of LabView platform. It achieves high accuracy with compact hardware and efficient algorithm, which will enlighten the future research in pathological mechanism of hearing loss.

\section{References}

[1] Deng J,Chen S. Using a Dynamic Tracking Filter to Extract Distortion product Otoacoustic Emissions Evoked with Swept-tone signals [J].IEEE Journal of Biomedical and Health information,2013

[2]Datian Ye, Cao Yu. Design and Test of Otoacoustic Emissions Detector [J]. Integration Technology, 2013(4).

[3]Jianqun Wan,Jinrui Nan. Using LabView to Complete a Data Collection System[J]. Computer Engineering and Application,2003(6).

[4]Siegel J, Hirohata E. Sound calibration and distortion product otoacoustic emission at high frequencies[J]. Hearing Research,1994,80:146-152. 